\title{
Analysis of codon usage bias of classical swine fever virus
}

\author{
Sharanagouda S. Patil(D, Uma Bharathi Indrabalan (D), Kuralayanapalya Puttahonnappa Suresh (iD and Bibek Ranjan Shome (D) \\ ICAR-National Institute of Veterinary Epidemiology and Disease Informatics (NIVEDI), Yelahanka, Bengaluru, \\ Karnataka, India. \\ Corresponding author: Sharanagouda S. Patil, e-mail: sharanspin13@gmail.com \\ Co-authors: UBI: balan.bharathi@yahoo.co.in, KPS: sureshkp97@rediffmail.com, BRS: brshome@gmail.com \\ Received: 12-01-2021, Accepted: 21-04-2021, Published online: 05-06-2021
}

doi: www.doi.org/10.14202/vetworld.2021.1450-1458 How to cite this article: Patil SS, Indrabalan UB, Suresh KP,

Shome BR (2021) Analysis of codon usage bias of classical swine fever virus, Veterinary World, 14(6): 1450-1458.

\begin{abstract}
Background and Aim: Classical swine fever (CSF), caused by CSF virus (CSFV), is a highly contagious disease in pigs causing $100 \%$ mortality in susceptible adult pigs and piglets. High mortality rate in pigs causes huge economic loss to pig farmers. CSFV has a positive-sense RNA genome of $12.3 \mathrm{~kb}$ in length flanked by untranslated regions at 5' and 3' end. The genome codes for a large polyprotein of 3900 amino acids coding for 11 viral proteins. The 1300 codons in the polyprotein are coded by different combinations of three nucleotides which help the infectious agent to evolve itself and adapt to the host environment. This study performed and employed various methods/techniques to estimate the changes occurring in the process of CSFV evolution by analyzing the codon usage pattern.
\end{abstract}

Materials and Methods: The evolution of viruses is widely studied by analyzing their nucleotides and coding regions/ codons using various methods. A total of 115 complete coding regions of CSFVs including one complete genome from our laboratory (MH734359) were included in this study and analysis was carried out using various methods in estimating codon usage bias and evolution. This study elaborates on the factors that influence the codon usage pattern.

Results: The effective number of codons (ENC) and relative synonymous codon usage showed the presence of codon usage bias. The mononucleotide (A) has a higher frequency compared to the other mononucleotides ( $\mathrm{G}, \mathrm{C}$, and T). The dinucleotides $\mathrm{CG}$ and $\mathrm{CC}$ are underrepresented and overrepresented. The codons CGT was underrepresented and AGG was overrepresented. The codon adaptation index value of 0.71 was obtained indicating that there is a similarity in the codon usage bias. The principal component analysis, ENC-plot, Neutrality plot, and Parity Rule 2 plot produced in this article indicate that the CSFV is influenced by the codon usage bias. The mutational pressure and natural selection are the important factors that influence the codon usage bias.

Conclusion: The study provides useful information on the codon usage analysis of CSFV and may be utilized to understand the host adaptation to virus environment and its evolution. Further, such findings help in new gene discovery, design of primers/probes, design of transgenes, determination of the origin of species, prediction of gene expression level, and gene function of CSFV. To the best of our knowledge, this is the first study on codon usage bias involving such a large number of complete CSFVs including one sequence of CSFV from India.

Keywords: classical swine fever virus, codon usage bias, India, nucleotide composition, synonymous codons.

\section{Introduction}

Classical swine fever (CSF) is caused by an enveloped RNA virus belonging to the family Flaviviridae of genus Pestivirus. It was found that the classical swine fever virus (CSFV) is antigenically related to the other pestiviruses such as bovine viral diarrhea virus of cattle, and border disease virus of sheep. CSFV is a highly prevalent and endemic disease, usually found affecting the swine. The infected pigs develop few symptoms such as diarrhea, nausea, fever, hemorrhages, stagnation, and discoloration seen in legs, ears, and abdomen. They might also develop neurological disorders, reproductive disorders, and usually abortions [1-3].

Copyright: Patil, et al. Open Access. This article is distributed under the terms of the Creative Commons Attribution 4.0 International License (http://creativecommons.org/licenses/by/4.0/), which permits unrestricted use, distribution, and reproduction in any medium, provided you give appropriate credit to the original author(s) and the source, provide a link to the Creative Commons license, and indicate if changes were made. The Creative Commons Public Domain Dedication waiver (http://creativecommons.org/ publicdomain/zero/1.0/) applies to the data made available in this article, unless otherwise stated.
The studies on analysis of codon usage pattern on CSFV are minimum or less. CSF is a very serious contagious disease found infecting different places around the world. The codon usage analysis is the most essential feature that plays a major role in biological evolution. The codon usage bias is found in the coding DNA, with difference in the frequencies of synonymous codons occurrences [4,5]. The synonymous codon is those which codes for the same amino acid, except for the codons that encode methionine and tryptophan. Some of the synonymous codons usage varies in different species, which is not random [5-7]. Natural selection, nucleotide base content, genetic mutation, and drift are some of the factors that are closely related to the codon bias in the molecular evolution of the agent/organisms. The codon usage experienced during the process of molecular evolution, is efficient in changing the production of proteins and mutations in the genes [5-9]. Therefore, codon usage analysis provides details on how it affects the evolution pattern, environmental adaptation, response 
to the immune system, and virus survival among the hosts and virus [7,10]. Further, analysis of codon usage bias is important in understanding the molecular biology, genetics, and genome evolution, it also helps in new gene discovery, design of primers, design of transgenes, determining the origin of species, and prediction of gene expression level and gene function.

Thus, the analysis on codon usage bias helps in obtaining an in-depth knowledge of mutations that leads to evolutionary changes and also to understand the changes in the viral adaptations. This study performed and employed various methods/techniques to estimate the changes occurring in the process of CSFV evolution by analyzing the codon usage pattern.

\section{Materials and Methods}

\section{Ethical approval}

Ethical approval is not applicable since the study used the data available in the public domain.

\section{Study period and location}

A total 115 complete CSFV sequences obtained from 1977 to 2019 from the GenBank (NCBI) were used in the study. The sequences were derived from 3 continents viz., Asia, Europe, and North America (Supplementary data can be available from the corresponding author).

\section{Sequence data retrieval}

One complete CSFV genome sequence from our laboratory (MH734359) [11], along with a total of 114 coding sequences (CDS) of CSFV from 20 different countries were retrieved from the GenBank database, National Center for Biotechnology Information (NCBI) (https://www.ncbi.nlm.nih.gov/nucleotide/). All the strains representing six subtypes (1.1, 1.2, 2.1, 2.2, 2.3, and 3.2) were used in this study and sequences with $>99 \%$ homogeneity were excluded (Supplementary data can be available from the corresponding author).

\section{Nucleotide composition analysis}

The whole-genome sequences of CSFV were aligned and edited using MEGA X (https://www.megasoftware.net/). The mononucleotide frequencies (A, $\mathrm{G}, \mathrm{C}$, and $\mathrm{T}$ ), the contents of $\mathrm{GC}$ at first, second, and third codon positions $\left(\mathrm{GC}_{1}, \mathrm{GC}_{2}\right.$, and $\left.\mathrm{GC}_{3}\right)$, and $\mathrm{GC}_{12}$ (mean of $\mathrm{GC}_{1}$ and $\mathrm{GC}_{2}$ ) were calculated using Seqinr library [12] in R software [13]. The frequencies of mononucleotides at the third position of synonymous codon $\left(\mathrm{A}_{3}, \mathrm{G}_{3}, \mathrm{C}_{3}\right.$, and $\left.\mathrm{T}_{3}\right)$ were obtained from MEGA X [14]. The index $\mathrm{GC}_{3}$ (at synonymous third codon position) was used to calculate the fraction of GC nucleotides at the synonymous third codon position (excluding Met [Methionine], Trp [Tryptophan], and the termination codons) [15]. These nucleotide parameters were used for further analysis to obtain codon usage analysis.

\section{Effective number of codons (ENC) and ENC-plot analysis}

The ENC values were used to enumerate the complete usage pattern of codon bias in the coding sequences (ORFs) and how it varies from the normal
Table-1: Frequencies of mononucleotides, GC contents, ENC and CAI of CSFV used in this study.

\begin{tabular}{lc}
\hline Mononucleotides & Frequency \\
\hline $\mathrm{A}$ & $0.31 \pm 0.04$ \\
$\mathrm{C}$ & $0.20 \pm 0.04$ \\
$\mathrm{G}$ & $0.26 \pm 0.04$ \\
$\mathrm{~T}$ & $0.21 \pm 0.04$ \\
$\mathrm{~A}_{3}$ & $0.28 \pm 0.03$ \\
$\mathrm{C}_{3}$ & $0.24 \pm 0.03$ \\
$\mathrm{G}_{3}$ & $0.27 \pm 0.03$ \\
$\mathrm{~T}_{3}$ & $0.19 \pm 0.03$ \\
\hline GC-contents & Frequency \\
\hline $\mathrm{GC}$ & $0.47 \pm 0.003$ \\
$\mathrm{GC}$ & $0.47 \pm 0.004$ \\
$\mathrm{GC}_{1}$ & $0.47 \pm 0.002$ \\
$\mathrm{GC}_{3}$ & $0.46 \pm 0.006$ \\
$\mathrm{GC}_{12}$ & $0.47 \pm 0.002$ \\
ENC value $_{\text {CAI value }}$ & $52.69 \pm 0.47$ \\
\hline $\mathrm{A}_{1} \mathrm{C}_{1} \mathrm{G}$, and & $0.71 \pm 0.003$ \\
\hline
\end{tabular}

A, C, G, and T denotes compositional frequency of A, C, G, and $T . A_{3}, C_{3}, G_{3}$ and $T_{3}$ denotes compositional frequency of $A_{3}, C_{3}, G_{3}$, and $T_{3}$ at third codon site, $G_{1}, G C_{2}, G C_{3}$ denotes the $\mathrm{GC}$ contents at first, second and third codon positions respectively. $\mathrm{GC}_{12}$ denotes mean of $\mathrm{GC}_{1}$ and $\mathrm{GC}_{2}$. ENC values represent the mean effective number of codons and CAI value represents the mean of Codon Adaptation Index of CSFV. CSFV=Classical swine fever virus, $\mathrm{CAI}=$ Codon adaptation index, ENC=Effective number of codons

usage of synonymous codons. ENC is considered as an estimator of codon usage bias in ORF. The values of ENC ranges from 20 to 61, indicating that the values closer to 20 preferred to have stronger codon usage bias whereas values nearer or equal to 35 have moderate codon usage bias and values closer to 60 have weaker codon usage bias. The ENC value is usually estimated with the following mathematical formula $[16,17]$ :

$$
\begin{gathered}
E N C^{C a l}=2+\frac{9}{f_{2}}+\frac{1}{f_{3}}+\frac{5}{f_{4}}+\frac{3}{f_{6}} \\
R S C U=\frac{g_{i j}}{\sum_{j}^{n_{i}} g_{i j}} n_{i}
\end{gathered}
$$

Where, $f_{2}, f_{3}, f_{4}$, and $f_{6}$ stands for values of $f_{i}$ for i-fold degenerate amino acids and the coefficients 9, 1,5 , and 3 indicate the different classes of amino acid. The $f_{i}$ is estimated with the formula [17]:

$$
f_{i}=\frac{m \sum_{k=1}^{i}\left(\frac{m_{k}}{m}\right)^{2}-1}{m-1}
$$

The total number of observed codons for the amino acid is represented as $\mathrm{m}$, the observed number of the $k$ th codon for the amino acid is represented as $m_{k}$. The coRdon library [18] in R software [15] was used to estimate the ENC values.

The relationship between $\mathrm{ENC}$ and $\mathrm{GC}_{3}$ value is mostly used to know how the codon usage patterns are influenced by several factors such as mutation pressure and natural selection. Therefore, ENC-plot analyzes the relationship between $\mathrm{ENC}$ and $\mathrm{GC}_{3}$ values. 
In the ENC-plot, the ENC values for every $\mathrm{GC}_{3}$ values were calculated with the formula as follows:

$$
E N C^{\exp }=2+s+\frac{29}{s^{2}+(1-s)^{2}}
$$

Where the $\mathrm{GC}_{3}$ values are denoted as $s$, and with the expected ENC values, a curve was produced. In the plot, if the observed ENC- $\mathrm{GC}_{3}$ values fall on the curve, it means that mutation was the main force acting on third position bases of codons whereas if observed ENC values fell considerably below the expected curve, it meant that selection was the main force driving codon usage bias [8-10]. If there is no natural selection, then evolution is mostly affected by mutational pressure. The codon usage would usually get affected by compositional parameters of the sequences. Therefore, the points are observed to fall on or near the expected ENC curve.

\section{Relative synonymous codon usage (RSCU) and prin-} cipal component analysis (PCA)

The RSCU values of each codon in each gene were used to measure codon usage. The RSCU value is the ratio of observed frequency value to the expected frequency value of the synonymous codons [10]. The RSCU values were calculated with the following formula:

$$
R S C U=\frac{g_{i j}}{\sum_{j}^{n_{i}} g_{i j}} n_{i}
$$

Where the observed number of the $i$ th codon for the $j$ th amino acid having $n_{i}$ synonymous codons. The RSCU values $>1$ represents codon abundance and have positive codon usage bias, whereas the RSCU values $<1$ represent less codon abundance and have negative codon usage bias. If the RSCU values are equal to 1 , then there is no codon usage bias. Further, if the RSCU values $>1.6$ represents overrepresented codons and $<0.6$ represents underrepresented codons [7-10]. The RSCU values were estimated using the Seqinr library of R software [12,19]

PCA is a dimensionality reduction technique that is mostly used to obtain the relationship between variables (RSCU) and their components (codons). To analyze the variants and dominant patterns in the usage of codons on coding sequences in CSFV, the PCA [20] was performed on the RSCU values except for the three stop codons and the two sense non-synonymous codons ATG and TGG. The 59 RSCU values for each sequence with their codons were taken for the PCA. The analysis was done using factoextra library [21] in R software. The factors that influenced codon usage bias were effectually validated with the analysis of PCA.

\section{Codon adaptation index (CAI)}

To measure the similarities in the usage of codon between the host and the virus, a CAI was performed. The CAI values range between 0 and 1; the higher CAI value indicates codon usage bias is higher and adaptive [22]. The CAI values were calculated using theDAMBE v7.2.1 software [23] with reference organism as Sus scrofa (pig). Those sequences with higher CAI values were chosen over the lower CAI values. It also indicates that the frequently used codons will preferably get adapted to their host [24].

\section{Neutral evolution analysis}

The neutrality evolution plot represents the influence of mutation pressure and natural selection effects on the codon usage bias. The neutral evolution is analyzed by plotting the regression line with the synonymous codons values of $\mathrm{GC}_{3}$ against $\mathrm{GC}_{12}[25,26]$. In this analysis, if the values are closer to one, they are statistically significant and the codon usage is mainly due to mutation pressure. If the slope is closer to zero, the selection is natural to codon usage bias. The linear regression analysis was performed using $\mathrm{R}$ software.

\section{Chargaff's second parity rule (PR2) analysis}

According to Chargaff's PR2, mononucleotides $\mathrm{A}=\mathrm{T}$ and $\mathrm{G}=\mathrm{C}$ in the coding sequences indicate that there is no bias in the selection and mutation. To evaluate the effect of mutation and natural selection pressure on the codon usage pattern, the PR2 is plotted with AT bias at third codon position $\left[\mathrm{A}_{3} /\left(\mathrm{A}_{3}+\mathrm{T}_{3}\right)\right]$ as ordinate against $\mathrm{GC}$ bias, at third codon position $\left[\mathrm{G}_{3} /\left(\mathrm{G}_{3}+\mathrm{T}_{3}\right)\right]$ as abscissa and the origin at $(0.5,0.5)$ where $\mathrm{A}=\mathrm{T}$ and $\mathrm{G}=\mathrm{C}$ points lying have no bias with no affect towards mutation pressure and natural selection [10,27]. It is observed that the preference is toward purine than pyrimidine when the value is $>0.5$. Moreover, the mononucleotides base $\mathrm{A}$ is preferred over base $\mathrm{T}$ and base $\mathrm{G}$ is preferred over base $\mathrm{C}$ [28]. The bias resulting from mutations and natural selection helps us to measure the degree of deviance from PR2 [29].

\section{Dinucleotide abundance frequency analysis}

Dinucleotide abundance frequency was performed to analyze the effect of dinucleotide frequencies on codon usage patterns. The frequencies of dinucleotides are considered overrepresented if the value is $>1.23$ and underrepresented if $<0.78$. The dinucleotide frequency is calculated with the formula as follows [30]:

$$
P_{X Y}=\frac{f_{x y}}{f_{y} f_{x}}
$$

Where the frequency of nucleotides $\mathrm{X}$ and $\mathrm{Y}$ is denoted as $f_{x}$ and $f_{y}$ respectively. The expected frequency of the dinucleotide $\mathrm{XY}$ is denoted as $f f_{x}$ and the observed frequency of dinucleotide $\mathrm{XY}$ is denoted as $f_{x y}$ [9].

\section{Results}

\section{Sequence data retrieval}

In this study, a total of $115 \mathrm{CSFV}$ coding sequences (complete genome), including one from our laboratory (MH734359) were downloaded from the GenBank database of NCBI (https://www.ncbi. nlm.nih.gov), with their accession numbers in FASTA format on October 21, 2020. In this study, all 115 CSFV coding sequences were included for the codon usage analysis. 


\section{Nucleotide compositional analysis of CSFV}

The nucleotide content of the sequences was calculated, the frequencies of $\mathrm{A}, \mathrm{C}, \mathrm{G}$, and $\mathrm{T}$ were $31.27 \%, 20.76 \%, 26.28 \%$, and $21.66 \%$, respectively, and the mean composition of nucleotide $\mathrm{A}$ is higher and nucleotide $\mathrm{C}$ is the least (Figure-1A). The codon composition at the third position $\mathrm{G}_{3}, \mathrm{C}_{3}, \mathrm{~A}_{3}$, and $\mathrm{T}_{3}$, was $27.61 \%, 24.89 \%, 28.00 \%$, and $19.48 \%$, respectively, and the composition of $\mathrm{A}_{3}$ was found higher than the other nucleotides (Figure-1B). The mean compositions of $\mathrm{GC}, \mathrm{GC}_{1}, \mathrm{GC}_{2}, \mathrm{GC}_{3}$, and $\mathrm{GC}_{12}$ were $0.470,0.474838,0.472834,0.464195$, and 0.473836 , respectively (Figure-1C). $\mathrm{GC}_{1}$ and $\mathrm{GC}_{2}$ are higher and almost equal, whereas $\mathrm{GC}_{3}$ is low compared to $\mathrm{GC}_{1}$ and $\mathrm{GC}_{2}$ (Table-1).

\section{Effective number of codons (ENC) and ENC plot anal- ysis of CSFV}

The ENC is an essential component to evaluate the codon usage pattern and plays a very significant role in codon usage bias. In this study, the ENC values of CSFV coding sequences were ranging from 51.86 to 53.45 , with 52.69 as the mean ENC value showing a low codon usage bias. These results indicate that all the ENC values of CSFV are very high, as every ENC value is usually $>55$. The codon usage bias in CSFV is high compared to other RNA viruses (Table-1).

To analyze the usage of synonymous codons, the ENC values were plotted against $\mathrm{GC}_{3}$ values. The scatter plot shows the relationship between ENC and $\mathrm{GC}_{3}$ values which range between 51.86 and 53.45 of all 115 CSFV sequences (Figure-2B). In the ENCplot it is seen that all the values fall inside and closer to the expected curve henceforth indicating that the selection pressure is influenced by codon usage bias in CSFV. These results indicate that the mutation in
$\mathrm{GC}_{3}$ may also influence the codon usage bias in these sequences (Figure-2A).

\section{RSCU of CSFV}

RSCU values are usually amino acid composition independent and are mainly used to compare the codon usage among the sequences. Among the 59 codons, 17 codons were mostly used. In these 17 codons, four (ATA, CCA, CAA, and TCA) codons were ending with $\mathrm{A} / \mathrm{T}$ and 13 (GCC, TGC, GAC, GAG, TTC, GGG, CAC, AAG, CTG, AAC, AGG, and GTG) codons were ending with $\mathrm{C} / \mathrm{G}$. ATA (1.60), CTG (1.64), AGG (2.86), and AGA (2.59) were seen overrepresented (>1.6) and CGT (0.09), GCG (0.38), ACG (0.43), and CTT (0.45) were seen underrepresented $(<0.6)$ (Table-2).

\section{Visualization of RSCU of CSFV}

The PCA was performed with the RSCU values of CSFV. PCA plot analysis showed the first principal component (72.4\%) and second principal component $(18 \%)$ of all the 59 synonymous codons variations in the RSCU values (Table-2). Only the most represented codons among 59 codons have been considered as components. The codon usage pattern was influenced by the evolution in the RSCU analysis. The codons ending with $\mathrm{A} / \mathrm{T}$ and $\mathrm{G} / \mathrm{C}$ might influence the selection and mutation pressure (Figure-3).

\section{CAI of CSFV}

To evaluate the impact of the virus in the host, an effective extent of codon usage bias in CSFV, the CAI was calculated using DAMBE v7.2.1 [23]. In this study, the average value of CAI in CSFV was found to be 0.71 and also falls between 0 and 1, indicating that the synonymous codons of CSFV are frequently used (Table-1). It evaluates the measure of natural selection

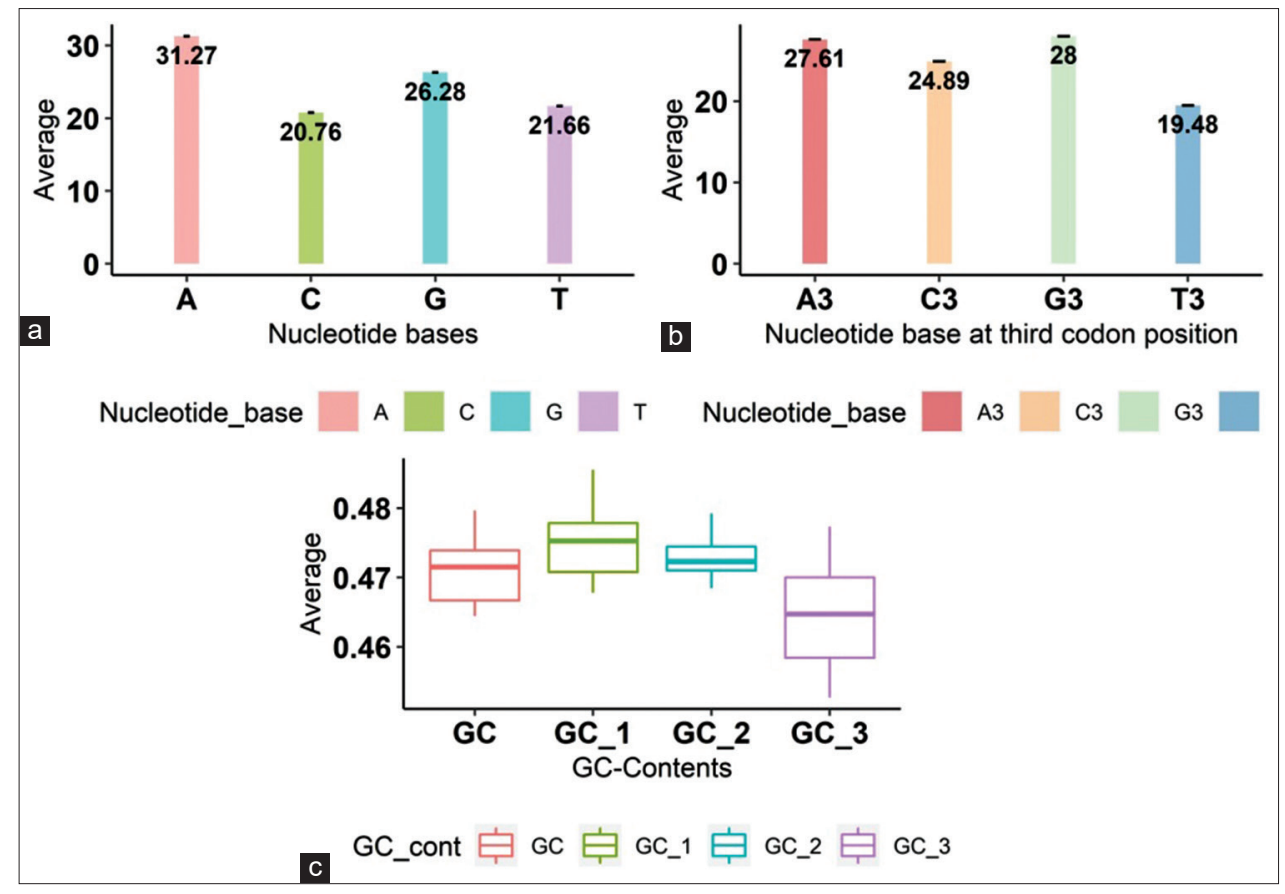

Figure-1: (a-c) Bar graphs showing nucleotide compositions of mononucleotides $(A, T, G, C)$, Mononucleotides $A_{3}, C_{3}$, ${ }_{3}$, and $T_{3}$ at third codon site, and GC contents of classical swine fever virus. 


\begin{tabular}{|c|c|c|c|c|c|}
\hline Amino acid & Codon & RSCU values & Amino acid & Codon & RSCU values \\
\hline \multirow[t]{2}{*}{ Phenylalanine } & $\pi T$ & $0.97 \pm 0.03$ & Serine & AGC & $1.64 \pm 0.11$ \\
\hline & TTC & $1.06 \pm 0.07$ & & AGT & $0.45 \pm 0.05$ \\
\hline \multirow[t]{6}{*}{ Leucine } & CTT & $1.02 \pm 0.03$ & & TCA & $0.97 \pm 0.02$ \\
\hline & CTC & $0.93 \pm 0.07$ & & TCC & $1.15 \pm 0.07$ \\
\hline & CTA & $1.29 \pm 0.10$ & & TCG & $1.02 \pm 0.02$ \\
\hline & CTG & $1.30 \pm 0.05$ & & TCT & $0.84 \pm 0.07$ \\
\hline & TTA & $0.43 \pm 0.10$ & Threonine & ACA & $1.36 \pm 0.05$ \\
\hline & TTG & $0.96 \pm 0.10$ & & ACC & $1.36 \pm 0.06$ \\
\hline \multirow[t]{3}{*}{ Isoleucine } & ATA & $2.59 \pm 0.09$ & & ACG & $0.38 \pm 0.04$ \\
\hline & ATC & $1.35 \pm 0.08$ & & ACT & $0.88 \pm 0.05$ \\
\hline & ATT & $2.86 \pm 0.10$ & Tyrosine & TAC & $0.87 \pm 0.09$ \\
\hline \multirow[t]{4}{*}{ Valine } & GTA & $1.41 \pm 0.09$ & & TAT & $0.86 \pm 0.04$ \\
\hline & GTC & $1.60 \pm 0.05$ & Glutamine & CAA & $1.39 \pm 0.05$ \\
\hline & GTG & $0.90 \pm 0.08$ & & CAG & $0.86 \pm 0.04$ \\
\hline & GTT & $0.48 \pm 0.11$ & Asparagine & AAC & $0.97 \pm 0.05$ \\
\hline \multirow[t]{4}{*}{ Proline } & CCA & $1.11 \pm 0.03$ & & AAT & $0.86 \pm 0.07$ \\
\hline & $\mathrm{CCC}$ & $1.12 \pm 0.08$ & Cysteine & TGC & $1.43 \pm 0.08$ \\
\hline & CCG & $0.88 \pm 0.03$ & & TGT & $0.72 \pm 0.11$ \\
\hline & CCT & $0.87 \pm 0.08$ & Histidine & CAC & $1.18 \pm 0.04$ \\
\hline \multirow[t]{4}{*}{ Alanine } & GCA & $1.47 \pm 0.07$ & & CAT & $0.81 \pm 0.04$ \\
\hline & GCC & $0.91 \pm 0.06$ & Arginine & AGA & $1.53 \pm 0.10$ \\
\hline & GCG & $0.64 \pm 0.08$ & & AGG & $0.73 \pm 0.07$ \\
\hline & $\mathrm{GCT}$ & $0.95 \pm 0.09$ & & CGA & $0.18 \pm 0.11$ \\
\hline \multirow[t]{4}{*}{ Glycine } & GGA & $0.12 \pm 0.04$ & & CGC & $0.76 \pm 0.09$ \\
\hline & GGC & $0.12 \pm 0.02$ & & CGG & $1.13 \pm 0.09$ \\
\hline & GGG & $0.19 \pm 0.04$ & & CGT & $0.86 \pm 0.09$ \\
\hline & GGT & $0.09 \pm 0.03$ & Aspartic acid & GAC & $0.77 \pm 0.05$ \\
\hline \multirow[t]{3}{*}{ Lysine } & AAA & $1.09 \pm 0.08$ & & GAT & $1.11 \pm 0.04$ \\
\hline & AAG & $0.72 \pm 0.04$ & Glutamic acid & GAA & $1.30 \pm 0.10$ \\
\hline & & & & GAG & $0.88 \pm 0.04$ \\
\hline
\end{tabular}

59 codons with corresponding amino acids of all 115 coding sequences of CSFV sequences, the values in bold represents the overrepresented codons in CSFV. CSFV=Classical swine fever virus, RSCU=Relative synonymous codon usage

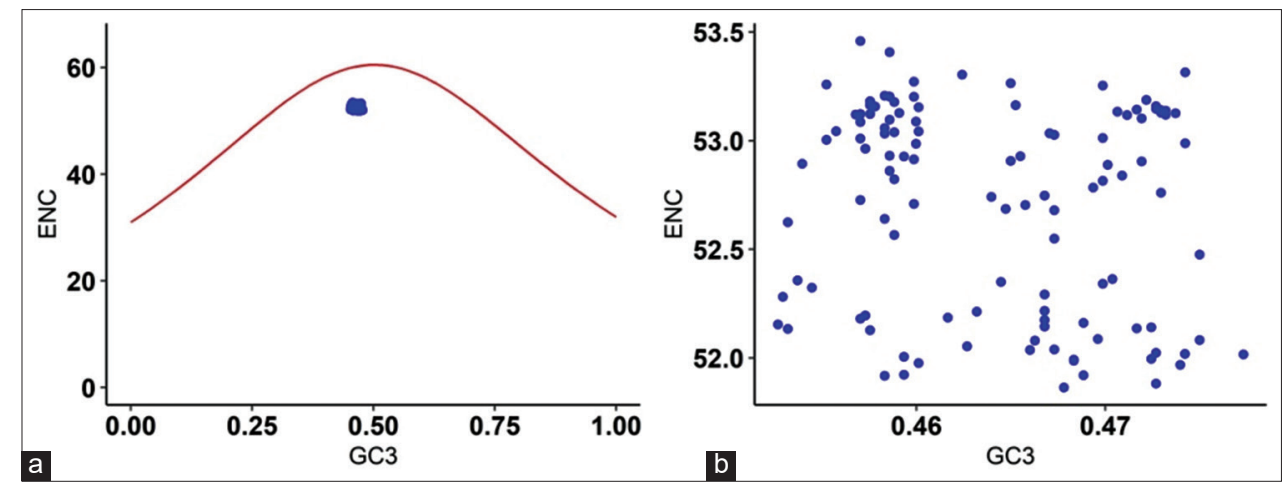

Figure-2: ( $a$ and b) Effective number of codons (ENC)-plot of classical swine fever virus (CSFV), the expected ENC curve plot represents ENC and $\mathrm{GC}_{3}$ values and scatter plot shows the relationship between ENC and $\mathrm{GC}_{3}$ values of all 115 CSFV sequences.

and how the codon usage bias is influenced among the CSFV sequences.

\section{Neutral evolution analysis of CSFV}

The neutrality plot was analyzed by plotting the values of $\mathrm{GC}_{3}$ against $\mathrm{GC}_{12}$; the plot was significant $\left(y=0.332+0.305 x, R^{2}=0.49\right)$ with $p<0.05$. The contents of $\mathrm{GC}_{12}$ and $\mathrm{GC}_{3}$ were varying slightly with an indication of low selection pressure; the codon usage pattern is influenced by GC contents of the nucleotides, and the natural selection contributed to the evolution of the codon usage pattern of CSFV (Figure-4).

\section{Chargaff's second PR2 analysis of CSFV}

To analyze the factors causing the codon usage bias in the CSFV, the PR2 bias, AT bias was plotted against $\mathrm{GC}$ bias, there was a minor deviation from the PR2 $(A=T$ and $C=G)$, whereas in the present study mononucleotide A was not equal to mononucleotide $\mathrm{T}$ and mononucleotide $\mathrm{G}$ was not equal to mononucleotide $\mathrm{C}$ in the third codon positions. In the PR2 plot, the distance between the values and the center indicates PR2 bias by its degree. Analysis revealed that AT and GC bias points were observed between 0.5 and 0.6 , indicating lower bias 


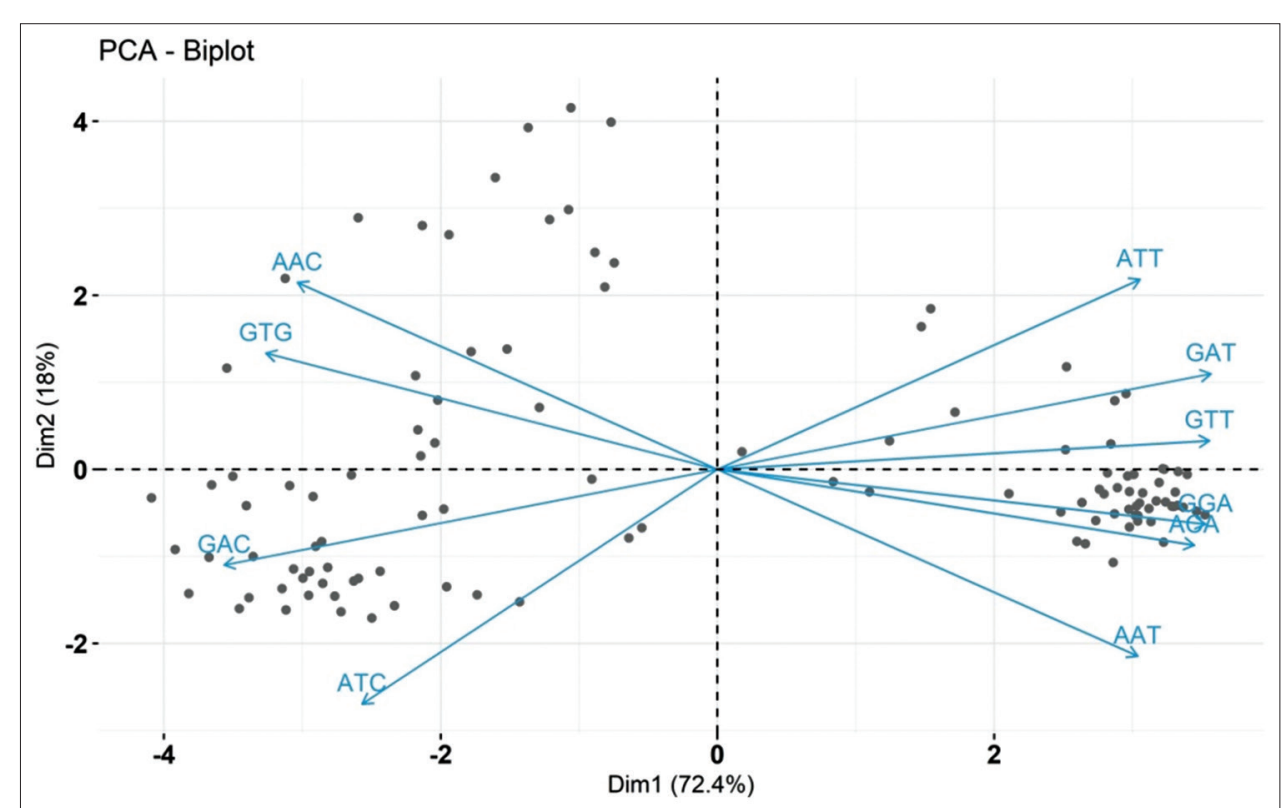

Figure-3: Principal component analysis (PCA) plot showing the deviations and similarity among the 59 codons of 115 classical swine fever virus sequences. PCA plot analysis showed the first principal component $(72.4 \%)$ and second principal component $(18 \%)$ of all the 59 synonymous codons variations in the relative synonymous codon usage values.

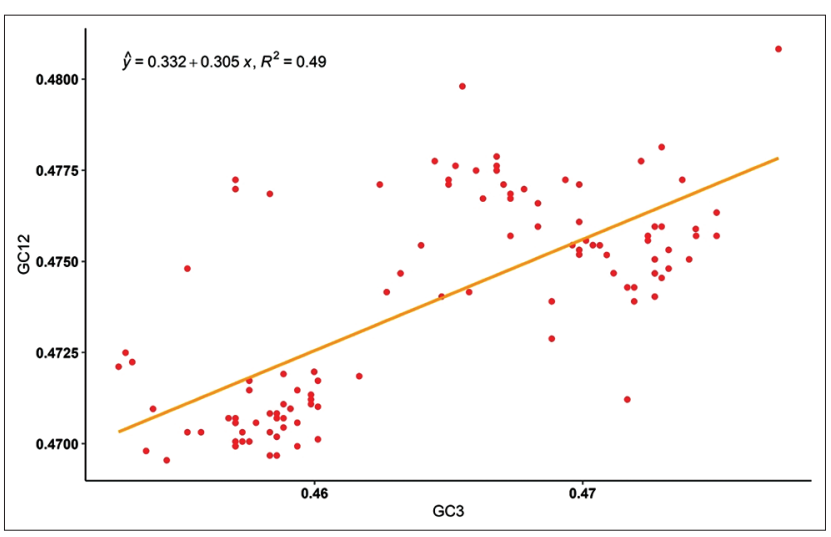

Figure-4: The neutrality plot analysis of classical swine fever virus (CSFV) sequences shows the correlation between the $\mathrm{GC}_{3}$ and $\mathrm{GC}_{12}$ and showing the influence of mutational bias in the CSFV.

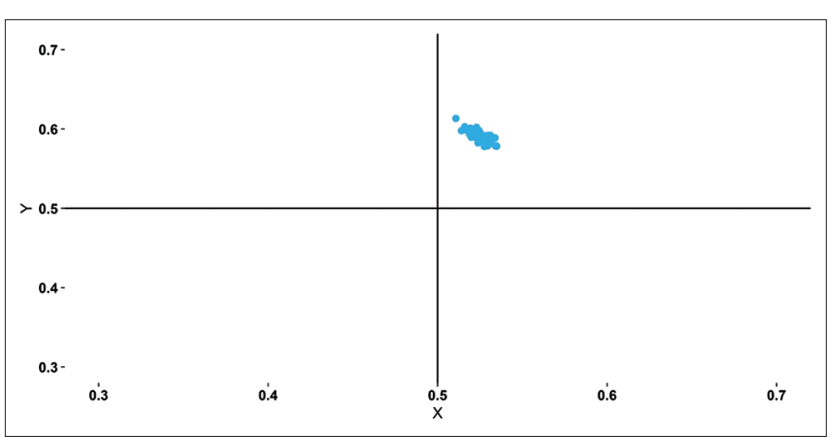

Figure-5: The PR2 plot was plotted using the obtained values with $X=G 3 /(G 3+C 3)$ and $Y=A 3 /(A 3+T 3)$ showing the mutation bias in classical swine fever virus.

(Figure-5). It was found that the mean AT bias was 0.52 and $\mathrm{GC}$ bias was 0.58 . Since the values are $>0.5$, A and $\mathrm{G}$ (Purines) are preferred over $\mathrm{T}$ and $\mathrm{C}$ (Pyrimidines).
Dinucleotide abundance frequency analysis of CSFV

Taking into view the abundant dinucleotide frequencies which affects the usage of codons, none of the dinucleotide frequency was equivalent to the estimated theoretic value $(=1.0)$, indicating that the dinucleotides frequencies values were varying. Among all the 16 dinucleotides, the frequency of dinucleotide CG $(0.430)$ was underrepresented $(\leq 0.78)$ whereas the frequencies of CC (1.250) and TG (1.240) were overrepresented ( $\geq 1.23)$, CT (1.209) was marginally overrepresented. The results show that the usage of codons was subjected to the abundant dinucleotide frequencies (Figure-6).

\section{Discussion}

In the present study, codon usage bias was analyzed using 115 complete coding sequences of CSFV, including one sequence from our laboratory. RNA viruses have their mutation rates higher and these rates are associated with the evolution and virulence factors to get adapted to the host environment. The mutation pressure, natural selection, frequencies of the mononucleotides, and $\mathrm{G} / \mathrm{C}$ content are the factors that are associated with the evolution of viruses and the usage of codons. The evolution of the virus is usually determined by the mononucleotides at the third codon position. The codon usage pattern usually tends to get affected by the varying nucleotide arrangement in the genome [5-10,26,31].

In this study, most of the codons in CSFV ORFs were found to be ending with $\mathrm{G}$ or $\mathrm{C}$. Mononucleotide $\mathrm{A}(31.27 \%)$ and $\left.\mathrm{A}_{3} 28.00 \%\right)$ at the third position were found to be higher compared to the other nucleotides in CSFV. The $\mathrm{GC}_{3}$ content was 0.46 which shows a small variation compared to $\mathrm{GC}_{1}$ and $\mathrm{GC}_{2}$. The variations 


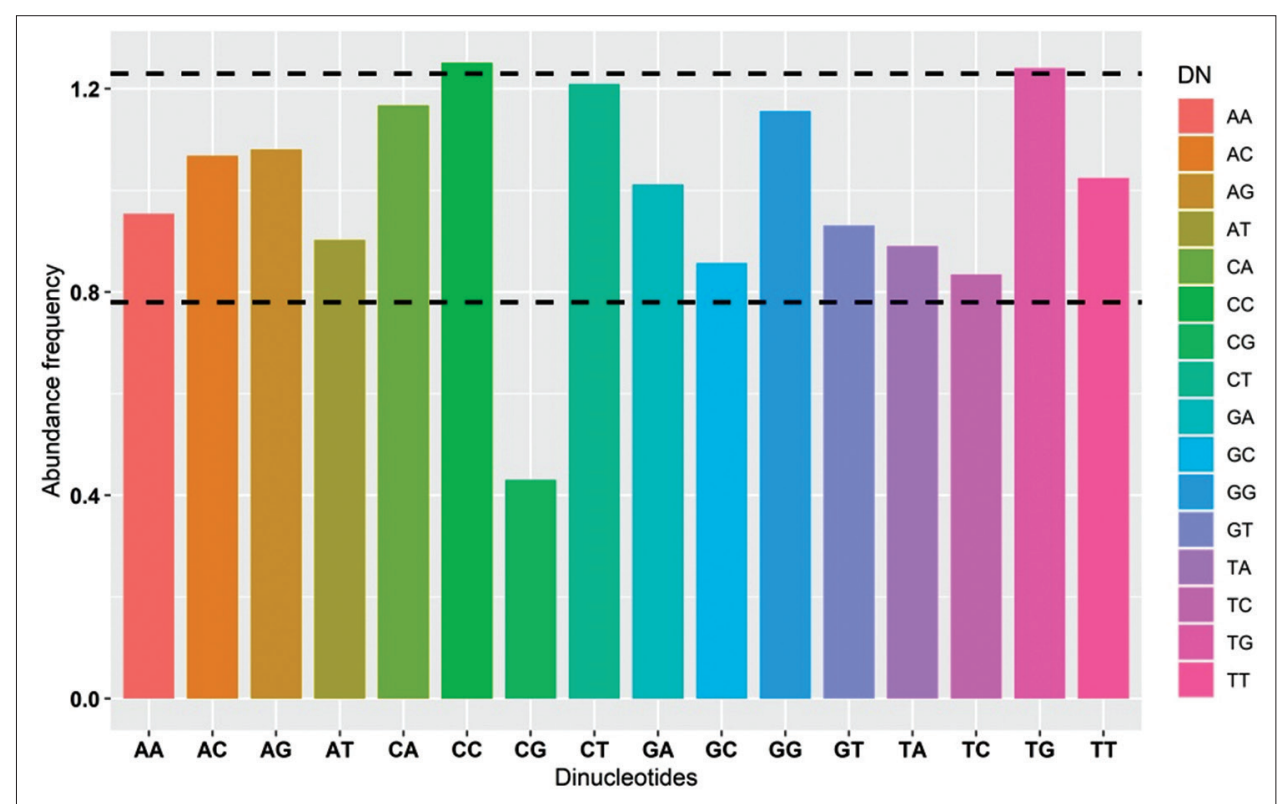

Figure-6: Dinucleotide abundance frequency of the classical swine fever virus (CSFV). The lines showing overrepresented and underrepresented values. The color variation represents 16 dinucleotides of CSFV.

in the base nucleotides and GC contents showed that there are mutations in the CSFV genome.

The RSCU values of 59 codons were calculated for $115 \mathrm{CSFVs}$ and the analysis indicated that the codons ending with $\mathrm{G}$ or $\mathrm{C}$ were abundant than those of $\mathrm{A}$ or $\mathrm{C}$ ending codons. The ATA, AGA, AGG, and CTG were overrepresented and ACG, CGT, CTT, and GCG were found to be underrepresented. Seventeen codons were found to have codon usage bias. AGG (2.86) was more overrepresented and GCT (0.09) was more underrepresented, whereas in [4] the AGG and GCT of CSFV were found to have 2.76 and 0.12 , respectively, indicating that there is less number of mutations. The variations among the synonymous codons were visualized by plotting the PCA plot which showed variation among the codons and are visible in the graph. Each axis in the PCA was 59 synonymous codons and the points in the PCA plot were the number of coding sequences, that is, 115 sequences used in this study.

The ENC values are very essential to obtain the codon usage bias and are considered to be very significant in codon usage analysis. The average ENC value was found to be $52.69 \pm 0.47$, indicating the low codon preference and a minimum bias of the codons. The mean ENC value for Atypical Porcine Pestivirus (APPV) was 54.62 \pm 0.09 [8], Porcine Astrovirus (PAstV) was 53.89 \pm 1.90 [10], and CSFV was $51.85 \pm 0.39$ obtained using 76 complete CSFV genomes [4]. On comparing with the above values, ENC values in the present study showed $52.69 \pm 0.47$ which is low for APPV and PAstV and high for CSFV [4]. It is conclusive that the overall codon usage bias is moderately less in this study. The ENC plot displays $\mathrm{GC}_{3}$ values against $\mathrm{ENC}$ values revealing the bias in usage of synonymous codons in CSFV. In the ENC plot, each point of ENC-GC ${ }_{3}$ is found lying below the expected ENC curve, indicating that the codon usage pattern was shaped by the mutation combined with natural selection. Although the ENC plot showed bias in the codon usage which was not so precise; hence, the analysis of the neutrality plot was carried out. This indicated that the rate of mutations in RNA viruses is significantly high.

The CAI values indicated that the nucleotide compositions and mutation pressure are the important factors affecting the codon usage pattern. The CAI values ranged from zero to one; the higher values (closer to 1) indicate that the usage of codons is similar and lower values indicate that the usage of codons is dissimilar (closer to 0). The CAI in this study was 0.71 , which revealed that there was a similarity in the codon usage.

In the analysis of the neutrality plot, the $\mathrm{GC}_{12}$ and $\mathrm{GC}_{3}$ correlated significantly, which infers that mutation pressure plays a significant role in the codon usage bias when compared to natural selection. Obtained R-squared value of 0.4905 and $\mathrm{p}<2.2 \mathrm{e}^{-16}$ showing that the plot is substantial. The PR2 plot was plotted using the obtained values with $\mathrm{A} 3 /(\mathrm{A} 3+\mathrm{T} 3)$ as ordinate and $\mathrm{G} 3 /(\mathrm{G} 3+\mathrm{C} 3)$ as abscissa [8]. It is seen that there is a codon usage bias visualizing the PR2 plot. The nucleotide $\mathrm{G}$ is not equal to nucleotide $\mathrm{C}(\mathrm{G} \neq \mathrm{C})$ and as nucleotide $\mathrm{A}$ is not equal to nucleotide $\mathrm{T}(\mathrm{A} \neq \mathrm{T})$, if there is no bias then nucleotide $\mathrm{A}$ is equal to nucleotide $\mathrm{T}$ $(A=T)$ and nucleotide $G$ will be equal to nucleotide $C$ $(G=C)[10,29]$. The analysis showed a codon usage inequity between AT and GC at the third codon base position, indicating that in addition to the mutation, the natural selection and/or good adaptation of the virus in pig population (hypothetically) might have affected the patterns of codon usage in CSFV $[32,33]$.

The frequencies of dinucleotides are affected by selection, mutation, and usage of the codons. In this 
study, the abundance of frequencies of 16 dinucleotides was obtained and plotted with frequencies as ordinate and the dinucleotides as abscissa. The colors in the graph were to differentiate the 16 different dinucleotides. The dinucleotide CG is underrepresented and dinucleotide TG and CC are overrepresented due to natural selection. The dinucleotide CG is usually underrepresented in most of the viruses $[30,31,34,35]$. The knowledge on codon usage bias in 115 CSFV genome obtained in this study would be of much needed in designing marker vaccine and vaccinology for CSF.

\section{Conclusion}

The synonymous codon usage of 115 complete coding sequences of CSFV has been analyzed. In the present study, it was observed that the codon usage pattern is directly influenced by compositions of mononucleotides, frequencies of dinucleotides, and GC content in CSFV. The study reveals that the evolution of the CSF virus was driven by the mutations in the codons. Evolutionary forces driving the evolution and diversity of CSFV is poorly understood. There are scanty reports on such studies using field isolates. It was shown in Cuban pig population that the vaccination under control program has led to positive selection on $\mathrm{B} / \mathrm{C}$ domain of the $\mathrm{E} 2$ protein for viral isolates circulating in Cuba (subgenotype 1.4) [36]. It was found that vaccination could affect CSFV diversity and might lead to the evasion of the immune response through recombination and point mutation, influencing the population dynamics, evolutionary rates, and adaptive evolution of CSFV [37,38]. Therefore, it is also possible that CSF viruses/strains while evading host immune mechanisms undergo evolution and diversity through recombination and point mutations. The present study undertaken was more focused on codon usage analysis using nucleotides and hence did not comment much on other methods of evolution. The analysis using various methods to study the codon usage bias of CSFV has been explained. Preferably, the codon usage bias observed here is due to the mutation in the nucleotides. The synonymous codon usage pattern and the dinucleotide frequencies are unique in CSFV. Hence, the evolution of CSFV might be due to mutation pressure combined with natural selection. To the best of our knowledge, this is the first report on codon usage bias and analysis of a large number of CSFV sequences, including the Indian strain of CSFV. Natural selection and mutation pressure are the main factors that influence the codon usage pattern. The information gained from this study will help researchers, academicians, and policymakers to apply such methodologies to various other livestock disease virus strains concerning to marker vaccines and vaccinology to study the evolution and codon usage of various viruses and their genetic evolution.

\section{Data availability}

Supplementary data can be available from the corresponding author on request.

\section{Author's Contribution}

SSP and KPS: Conceptualized and designed the study. SSP, KPS, and UBI: Conducted the analyses and interpreted the results. UBI and KPS: Drafted the manuscript. BRS: Edited the manuscript. All authors revised, edited, read, and approved the manuscript.

\section{Acknowledgments}

The authors thank DG, DDG (AS) ICAR, New Delhi, and the Director of the ICAR-NIVEDI for providing necessary funds, infrastructure facility, and guidance throughout the study. The study was funded by the ICAR-NIVEDI under Project Id: IXX08329.

\section{Competing Interests}

The authors declare that they have no competing interests.

\section{Publisher's Note}

Veterinary World remains neutral with regard to jurisdictional claims in published institutional affiliation.

\section{References}

1. Edwards, S., Fukusho, A., Lefevre, P.C., Lipowski, A., Pejsak, Z., Roehe, P. and Westergaard, J. (2000) Classical swine fever: The global situation. Vet. Microbiol., 73(2-3): 103-119.

2. Patil, S.S., Hemadri, D., Shankar, B.P., Raghavendra, A., Veeresh, H., Sindhoora, B., Chandan, S., Sreekala, K., Gajendragad, M.R. and Prabhudas, K. (2010) Genetic typing of recent classical swine fever isolates from India. Vet. Microbiol., 141(3-4): 367-373.

3. Patil, S.S., Hemadri, D., Veeresh, H., Sreekala, K., Gajendragad, M.R. and Prabhudas, K. (2012) Phylogenetic analysis of NS5B gene of classical swine fever virus isolates indicate plausible Chinese origin of Indian subgroup 2.2 viruses. Virus Genes, 44(1): 104-108.

4. Xu, X., Fei, D., Han, H., Liu, H., Zhang, J., Zhou, Y., Xu, C., Wang, H., Cao, H. and Zhang, H. (2017) Comparative characterization analysis of synonymous codon usage bias in classical swine fever virus. Microb. Pathog., 107(6): 368-371.

5. Tao, P., Dai, L., Luo, M., Tang, F., Tien, P. and Pan, Z. (2009) Analysis of synonymous codon usage in classical swine fever virus. Virus Genes, 38(1): 104-112.

6. Zhang, H., Leng, C., Tian, Z., Liu, C., Chen, J., Bai, Y., Li, Z., Xiang, L., Zhai, H., Wang, Q., Peng, J., An, T., Kan, Y., Yao, L., Yang, X., Cai, X. and Tong, G. (2018) Complete genomic characteristics and pathogenic analysis of the newly emerged classical swine fever virus in China. BMC Vet. Res., 14(1): 204.

7. Guan, D.L., Ma, L.B., Khan, M.S., Zhang, X.X., Xu, S.Q. and Xie, J.Y. (2018) Analysis of codon usage patterns in Hirudinaria manillensis reveals a preference for GC-ending codons caused by dominant selection constraints. $B M C$ Genomics, 19(1): 542.

8. Pan, S., Mou, C., Wu, H. and Chen, Z. (2020) Phylogenetic and codon usage analysis of atypical porcine pestivirus (APPV). Virulence, 11(1): 916-926.

9. Wang, X., Xu, W., Fan, K., Chiu, H.C. and Huang, C. (2020) Codon usage bias in the $\mathrm{H}$ gene of canine distemper virus. Microb. Pathogen., 149(12): 104511.

10. Wu, H., Bao, Z., Mou, C., Chen, Z. and Zhao, J. (2020) Comprehensive analysis of codon usage on porcine astrovirus. Viruses., 12(9): 991.

11. Patil, S.S., Suresh, K.P., Amachawadi, R.G., Meekins, D.A., 
Richt, J.A., Mondal, M., Hiremath, J., Hemadri, D., Rahman, H. and Roy, P. (2019) Genome sequence of classical swine fever virus NIVEDI-165, subtype 1.1, a field virus strain isolated from the Southern part of India. Microbiol. Resour. Announc., 8(21): e00295-19.

12. Charif, D. and Lobry, J.R. (2007) SeqinR 1.0-2: A contributed package to the $\mathrm{R}$ project for statistical computing devoted to biological sequences retrieval and analysis. In: Structural Approaches to Sequence Evolution. Springer, Berlin, Heidelberg. p207-232.

13. Team, R.C. (2020) R: A Language and Environment for Statistical Computing. R Foundation for Statistical Computing, Vienna, Austria.

14. Kumar, S., Stecher, G., Li, M., Knyaz, C. and Tamura, K. (2018) MEGA X: Molecular evolutionary genetics analysis across computing platforms. Mol. Biol. Evol., 35(6): $1547-1549$.

15. Ahmad, T., Sablok, G., Tatarinova, T.V., Xu, Q., Deng, X. and Guo, W. (2013) Evaluation of codon biology in citrus and Poncirus trifoliata based on genomic features and frame corrected expressed sequence tags. DNA Res., 20(2): $135-150$.

16. Wright, F. (1990) The effective number of codons used in a gene. Gene, 87(1): 23-29.

17. Fuglsang, A. (2004) The effective number of codons revisited. Biochem. Biophys. Res. Commun., 317(3): 957-964.

18. Elek, A., Kuzman, M. and Vlahoviček, K. (2020) coRdon: Codon usage analysis and prediction of gene expressivity. Bioconductor, 3(3): 11.

19. Sharp, P.M. and Li, W.H. (1986) An evolutionary perspective on synonymous codon usage in unicellular organisms. J. Mol. Evol., 24(1-2): 28-38.

20. Wold, S., Esbensen, K. and Geladi, P. (1987) Principal component analysis. Chemometr. Int. Lab. Syst., 2(1-3): 37-52.

21. Kassambara, A. and Mundt, F. (2020). factoextra: Extract and visualize the results of multivariate data analyses (Version R Package version 1.0.7.) [Computer software, USA]. Available from: https://rpkgs.datanovia.com/factoextra/index.html. Retrieved on 01-06-2021.

22. Gun, L., Yumiao, R., Haixian, P. and Liang, Z. (2018) Comprehensive analysis and comparison on the codon usage pattern of whole Mycobacterium tuberculosis coding genome from different areas. BioMed Res. Int., 2018(5): 574976.

23. Xia, X. (2018) DAMBE7: New and improved tools for data analysis in molecular biology and evolution. Mol. Biol. Evol., 35(6): 1550-1552.

24. Nasrullah, I., Butt, A.M., Tahir, S., Idrees, M. and Tong, Y. (2015) Genomic analysis of codon usage shows the influence of mutation pressure, natural selection, and host features on Marburg virus evolution. BMC Evol. Biol., 15(1): 174 .

25. Sueoka, N. (1988) Directional mutation pressure and neutral molecular evolution. Proc. Natl. Acad. Sci., 85(8): 2653-2657.

26. Deb, B., Uddin, A. and Chakraborty, S. (2020) Codon usage pattern and its influencing factors in different genomes of hepadnaviruses. Arch. Virol., 165(3): 557-570.

27. Sueoka, N. (1995) Intrastrand parity rules of DNA base composition and usage biases of synonymous codons. $J$. Mol. Evol., 40(3): 318-325.

28. Khandia, R., Singhal, S., Kumar, U., Ansari, A., Tiwari, R., Dhama, K., Das, J., Munjal, A. and Singh, R.K. (2019) Analysis of Nipah virus codon usage and adaptation to hosts. Front. Microbiol., 10(5): 886.

29. Wang, L., Xing, H., Yuan, Y., Wang, X., Saeed, M., Tao, J., Feng, W., Zhang, G., Song, X. and Sun, X. (2018) Genomewide analysis of codon usage bias in four sequenced cotton species. PLoS One., 13(3): e0194372.

30. Kariin, S. and Burge, C. (1995) Dinucleotide relative abundance extremes: A genomic signature. Trends Genet., 11(7): 283-290.

31. Yao, X., Fan, Q., Yao, B., Lu, P., Rahman, S.U., Chen, D. and Tao, S. (2020) Codon usage bias analysis of bluetongue virus causing livestock infection. Front. Microbiol., 11(5): 655 .

32. Rudner, R., Karkas, J.D. and Chargaff, E. (1968) Separation of B. subtilis DNA into complementary strands. 3. Direct analysis. Proc. Natl. Acad. Sci. U. S. A., 60(3): 921-922.

33. Karumathil, S., Raveendran, N.T., Ganesh, D., Sampath Kumar, N.S., Nair, R.R. and Dirisala, V.R. (2018) Evolution of synonymous codon usage bias in West African and Central African strains of monkeypox virus. Evol. Bioinform. Online, 14(3): 1-22.

34. Drake, J.W. and Holland, J.J. (1999) Mutation rates among RNA viruses. Proc. Natl. Acad. Sci. U. S. A., 96(24): 13910-13913.

35. Karlin, S., Doerfler, W. and Cardon, L.R. (1994) Why is CpG suppressed in the genomes of virtually all small eukaryotic viruses but not in those of large eukaryotic viruses? J. Virol., 68(5): 2889-2897.

36. Perez, L.J., de Arce, H.D., Perera, C.L., Rosell, R., Frías, M.T., Percedo, M.I., Tarradas, J., Dominguez, P., Núñez, J.I. and Ganges, L. (2012) Positive selection pressure on the $\mathrm{B} / \mathrm{C}$ domains of the $\mathrm{E} 2$-gene of classical swine fever virus in endemic areas under $\mathrm{C}$-strain vaccination. Infection, genetics and evolution. Infect. Genet. Dis., 12(7): 1405-1412.

37. Ji, W., Niu, D.D., Si, H.L., Ding, N.Z. and He, C.Q. (2014) Vaccination influences the evolution of classical swine fever virus. Infection, genetics and evolution. Infect. Genet. Dis., 25(7): 69-77.

38. Hu, D., Lv, L., Gu, J., Chen, T., Xiao, Y. and Liu, S. (2016) Genetic diversity and positive selection analysis of classical swine fever virus envelope protein gene E2 in east china under C-strain vaccination. Front. Microbiol., 7(2): 85. 